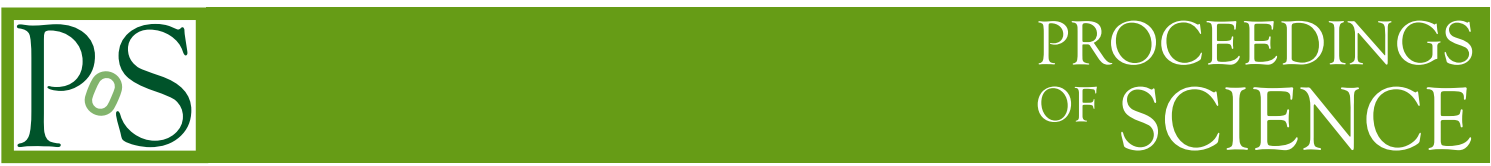

\title{
Design and performance of the future cluster-jet target for PANDA at FAIR
}

\author{
Esperanza Köhler*† \\ Institut für Kernphysik, Westfälische Wilhelms-Universität Münster \\ E-mail: e_koeh02@uni-muenster.de
}

\begin{abstract}
The internal cluster-jet target will be one of the two target stations for the planned PANDA experiment at the antiproton accelerator and storage ring HESR/FAIR. Due to the significance of investigations of antiproton-nucleon interactions for PANDA, hydrogen and deuterium are of highest interest as target material. This type of target allows for a high and constant target density at the interaction point as well as for the possibility to vary the target density continuously during operation. The prototype of the cluster-jet target has been built up in complete $\bar{P} A N D A$ geometry and set into operation at the university of Münster. Using this prototype, important information on the future target properties such as target beam dimensions and absolute target thickness at the interaction point, i.e. $2 \mathrm{~m}$ behind the nozzle, can be gained directly. The design concept of the cluster generator for $\overline{\mathrm{P}} \mathrm{ANDA}$ and the achieved performance will be presented.
\end{abstract}

8th International Conference on Nuclear Physics at Storage Rings-Stori11,

October 9-14, 2011

Laboratori Nazionali di Frascati dell'INFN, Italy

\footnotetext{
${ }^{*}$ Speaker.

${ }^{\dagger}$ Supported by EU (FP 6 and FP7), BMBF (06MS253 and 06MS9149I) and GSI F+E
} 
An internal target has to fulfil several challenging requirements. The used target material has to be of high purity to prevent background reactions. Furthermore the target thickness should be homogeneous and constant in time to avoid luminosity fluctuations at the data acquisition. In dependence of the experimental programme the target density, beam shape and size have to be variable. Especially the effective target beam size should be as small as possible for low influences on the vacuum conditions at the storage ring. The most challenging requirement at $\bar{P} A N D A$ is the distance of $2.1 \mathrm{~m}$ of the target source from the interaction point for a full $4 \pi$ solid angle acceptance. In addition an areal thickness in order of $10^{15}$ atoms $/ \mathrm{cm}^{2}$ at the interaction point is required to fully exploit the antiproton production rate. A high density cluster-jet target will be one of two planned target stations for the PANDA-experiment (see also [1]).

\section{Overview of the high density cluster-jet target prototype for $\overline{\mathbf{P}} \mathrm{ANDA}$}

A cluster-jet target prototype was designed, built up and set successfully into operation at the university of Münster. The complete system is installed in complete PANDA geometry, so that the observed cluster beam characteristics can be directly transferred to the situation at $\bar{P} A N D A$. The prototype consists of a cluster source, a scattering chamber and a beam dump, which are shown at Fig.1. The scattering chamber corresponds to the $\bar{P} A N D A$ interaction point in the required distance of $2.1 \mathrm{~m}$ from the target source.

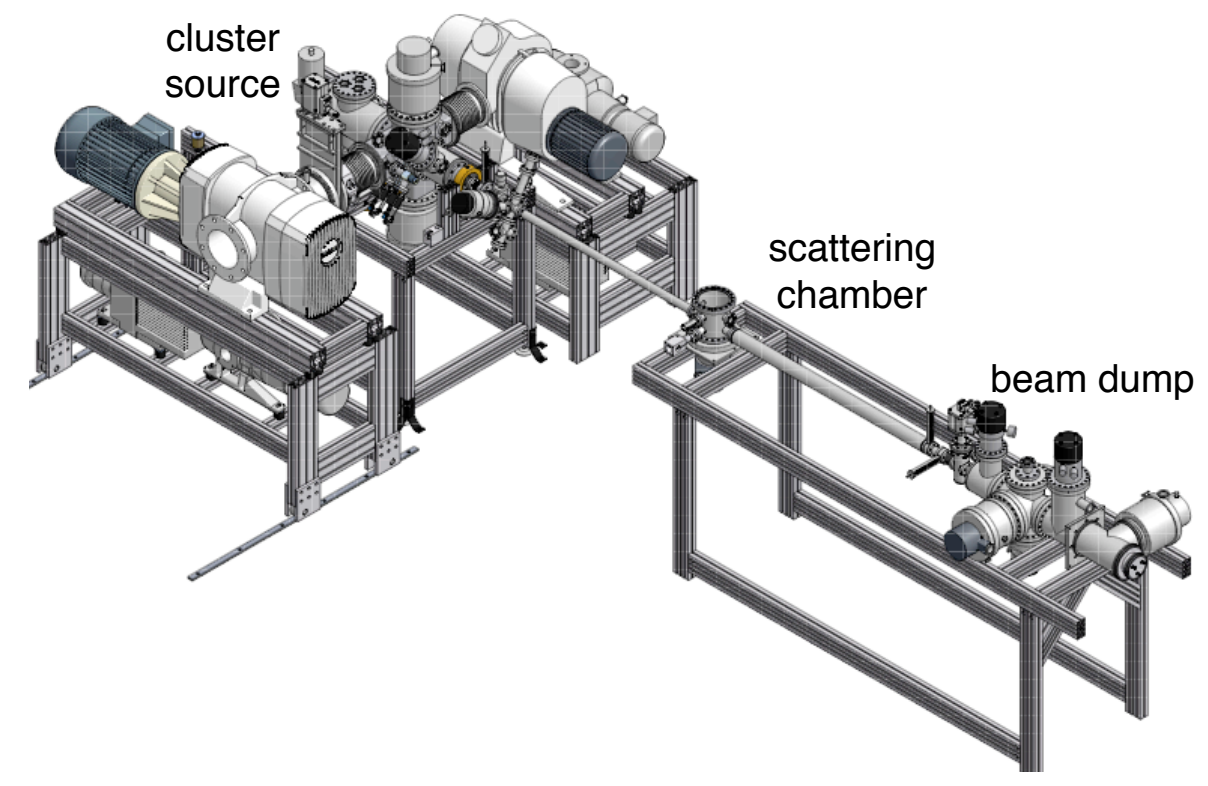

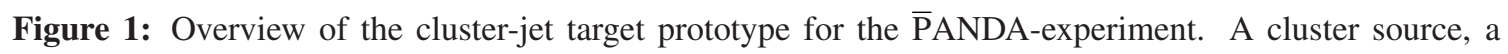
scattering chamber and a beam dump are the main parts of the setup. The scattering chamber corresponds to the interaction point at a distance of $2.1 \mathrm{~m}$ from the target source 
Hydrogen with a purity level of 9.0 is used mainly as target material, but the operation of deuterium or other target materials is also possible. Clusters are formed by the expansion of cooled hydrogen through a Laval nozzle into a vacuum chamber. The cold head, which cools the gas pipes and the hydrogen, is embedded in an insulation vacuum chamber as one can see at Fig. 2. At the narrowest point the used nozzle has an inner diameter of approximately $28 \mu \mathrm{m}$. Typical temperature and pressure settings are in the range of $19-50 \mathrm{~K}$ and $7-20$ bar. Only a small part of the hydrogen forms to clusters, so a differential pump system is used to separate the residual gas from the target beam. At the first vacuum chamber, the skimmer chamber, a skimmer extract just a small part of the cluster beam into another vacuum chamber. In the second vacuum chamber a second skimmer, the collimator, further cuts the cluster beam and defines the beam size and shape at the interaction point. Skimmer and collimator are both installed on X-Y-tables and movable $( \pm 2 \mathrm{~mm})$ during operation for final adjustments.

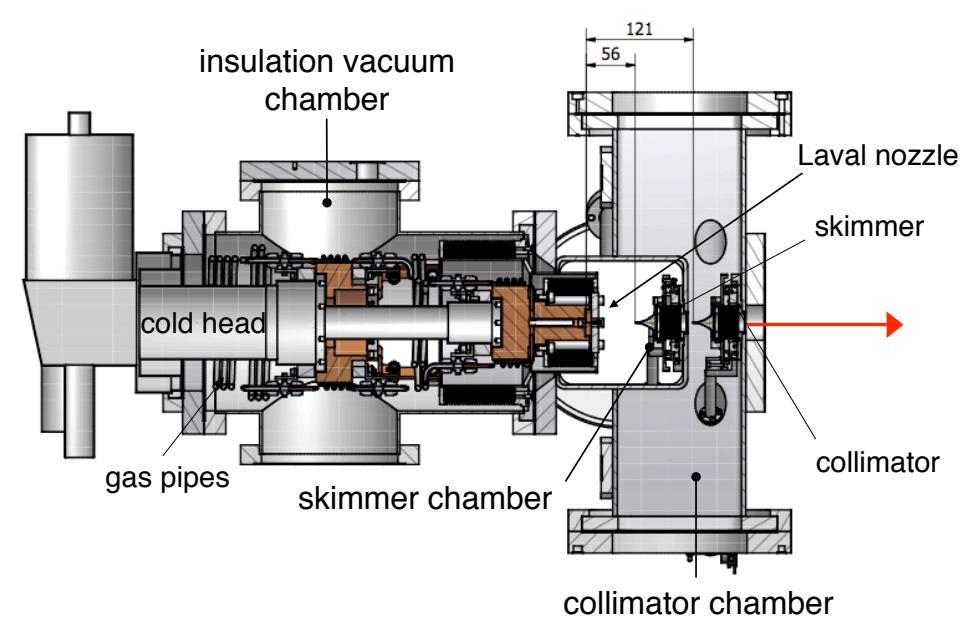

Figure 2: Overview of the cluster-jet source and its differential pump system. The cold head, which cools the hydrogen, is embedded in an insulation vacuum chamber. During the expansion of hydrogen through the Laval nozzle clusters are formed and injected into the skimmer chamber. There a skimmer extracts a part of the cluster beam into the collimator chamber, where a second skimmer, the collimator, defines the beam size and shape at the interaction point. Skimmer and collimator are installed in a distance of $56 \mathrm{~mm}$ and $121 \mathrm{~mm}$ from the narrowest point of the nozzle. The red arrow indicates the direction of the cluster beam.

The scattering chamber is installed in a distance of about two metres from the nozzle and is equipped with a beam diagnostic system shown at Fig. 3. This system works with two movable rods with a diameter of $1 \mathrm{~mm}$, which allow to scan the cluster beam in vertical and horizontal direction. At every step of the rods the pressure increase in the scattering chamber is recorded. With the measured beam profile, which is also presented in Fig. 3, it is possible to determine the cluster beam position, size and also the target density.

The last part of the target system represents the cluster beam dump, a differential pump system, where different pump types are installed. 

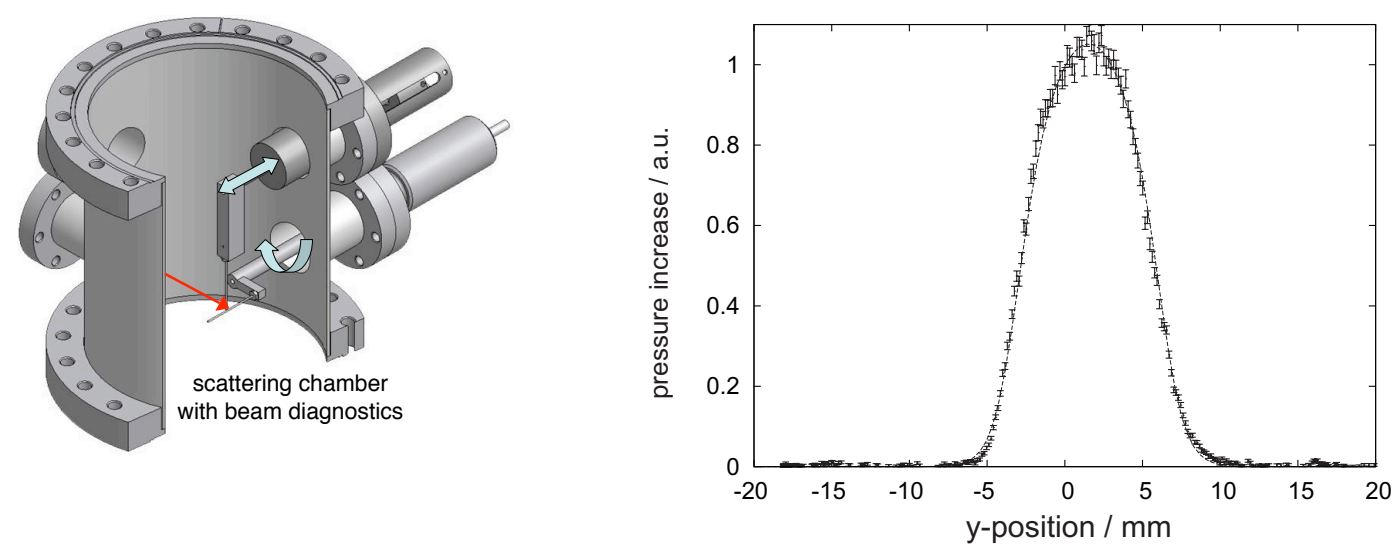

Figure 3: Left: Scattering chamber with beam diagnostic system, Right: Measured cluster beam profile at the interaction point. The target density, beam position and size can be determined by the pressure increase, the y-position and the observed width of the profile.

\section{Improvement of target density}

The measured cluster velocity, in the range of $200-1000 \mathrm{~m} / \mathrm{s}$ (see also [2], [3]), and the pressure increase in the scattering chamber are important variables for the determination of the target density. In Tab. 1 important parameters of different cluster-jet targets are presented. Remarkable is that the cluster-jet target prototype for $\overline{\mathrm{P} A N D A}$ is working at the largest distance $r$. Although the target density decreases with $1 / r^{2}$ the current setup achieves a max. target thickness of up to $10^{15} \mathrm{~cm}^{-2}$.

\begin{tabular}{c|c|c|c|c|} 
& $\begin{array}{c}\text { PROMISE/WASA } \\
\text { (CELSIUS) }\end{array}$ & $\begin{array}{c}\text { E835 } \\
\text { (FERMILAB) }\end{array}$ & $\begin{array}{c}\text { ANKE, COSY11 } \\
\text { (COSY) }\end{array}$ & $\begin{array}{c}\text { PANDA Prototype } \\
\text { (IKP Münster) }\end{array}$ \\
\hline $\begin{array}{c}\text { nozzle diameter } \\
\text { gas temperature }\end{array}$ & $20-35 \mathrm{~K}$ & $15-40 \mathrm{~K}$ & $22-35 \mathrm{~K}$ & $19-35 \mathrm{~K}$ \\
$\begin{array}{c}\text { gas pressure } \\
\text { distance } \\
\text { from nozzle } r\end{array}$ & $1.4 \mathrm{bar}$ & $<8 \mathrm{bar}$ & $18 \mathrm{bar}$ & $>18 \mathrm{bar}$ \\
$\begin{array}{c}\text { max. } \\
\text { target thickness }\end{array}$ & $1.3 \times 10^{14} \mathrm{~cm}^{-2}$ & $2 \times 10^{14} \mathrm{~cm}^{-2}$ & $\gg 10^{14} \mathrm{~cm}^{-2}$ & $\geq 10^{15} \mathrm{~cm}^{-2}$
\end{tabular}

Table 1: Important parameters of different cluster-jet targets. The max. target thickness of up to $10^{15} \mathrm{~cm}^{-2}$ was achieved with the current setup at a distance of $2.1 \mathrm{~m}$ from the interaction point. 
A measurement series above the critical point of the hydrogen vapour-pressure curve at $17 \mathrm{bar}$ shows the volume density and the areal target density, in dependence of the temperature. As in Fig. 4 presented the target density is easy to vary over several orders of magnitude. Furthermore one can observe an increase of the target density with decreasing temperature up to $24 \mathrm{~K}$ with small steep steps, e.g. at $35 \mathrm{~K}$, are due to different phases at the cluster formation process. The most striking point here is an unexpected decrease of the target density below $24 \mathrm{~K}$.

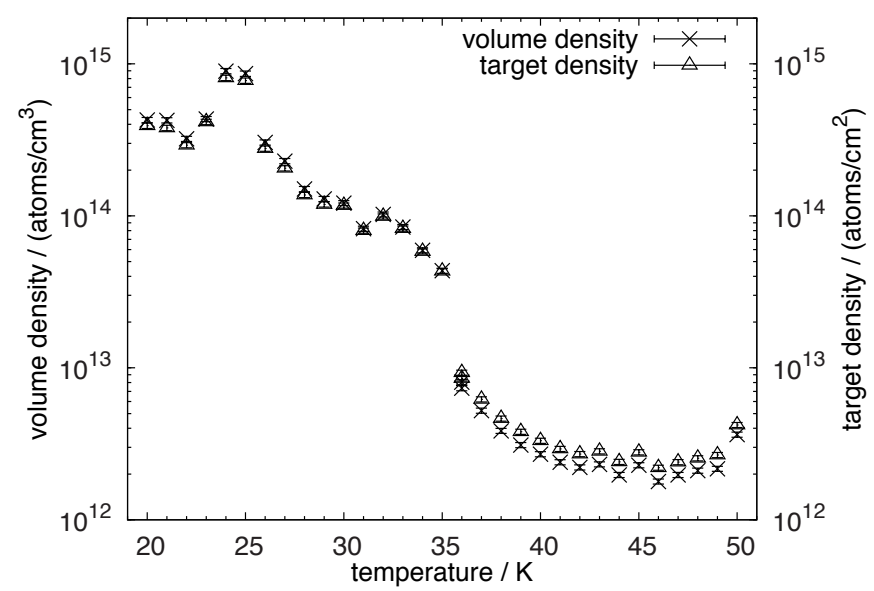

Figure 4: Volume density or target thickness in dependence of the temperature at 17 bar [4]. The target density is easy to vary over several orders of magnitude.

In order to investigate this effect the cluster beam in the skimmer chamber has been studied. A camera and a diode laser for illumination of the cluster-jet were installed. In Fig. 5 photographs of the cluster beam in the skimmer chamber are shown.
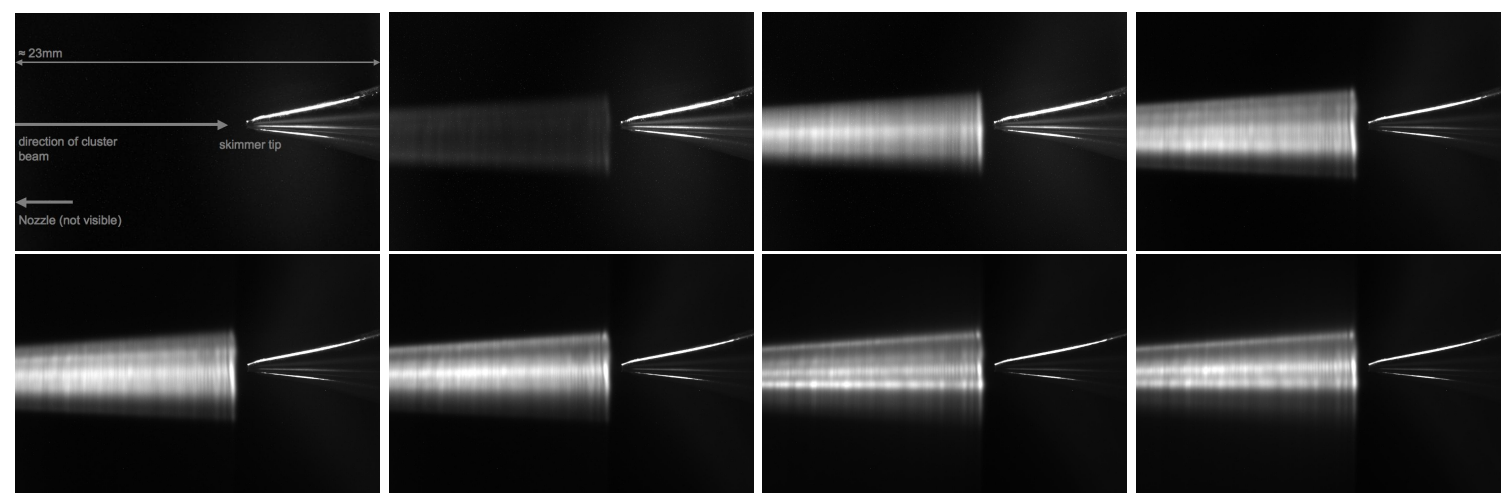

Figure 5: Photographs of the cluster beam in the skimmer chamber at a constant hydrogen pressure of 18.5 bar at 50, 37, 35, 30, 27, 25, 20 and $19 \mathrm{~K}$. The cluster beam leaves the nozzle exit (not visible) from the right and hits the skimmer (left). Due to practical reasons only the left part of the cluster beam was illuminated. At $50 \mathrm{~K}$ the cluster beam is not yet visible. The appearance of the cluster beam changes with each temperature variation. 
Each picture corresponds to a horizontal width of $23 \mathrm{~mm}$. Clearly visible is the skimmer tip on the right and the direction of the cluster beam is from left to the right. The photographs show an inhomogeneous cluster beam at 37, 35, 30, 27, 25, 20 and $19 \mathrm{~K}$, which changes its appearance with every change of the temperature (see also [5]). The density at the interaction point is still constant, because the skimmer extracts just a small part of the beam. This also confirms the measured beam profiles. To measure the density of the bright and less bright parts of the cluster beam a movable nozzle is required.

For this reason a tilting system consisting of a spherical joint and a nozzle holder extension was designed. The spherical joint is installed between the vacuum chamber of the cold head and the skimmer chamber. To guarantee the same position of the nozzle, a nozzle extension is also used. With this tilting system it is possible to adjust angles up to $\pm 5^{\circ}$ with the narrowest part of the nozzle as center of rotation.

The observation of the cluster beam in the skimmer chamber with the tilting system in operation is shown in Fig. 6. One can see that the angle of the cluster beam can be varied and as consequence the target density changes at constant hydrogen pressure and temperature settings. The decreasing target density and the variations are because of the structure of the cluster beam and the extraction of a less bright part. In first tests with the tilting system a volume density of up to $1.9 \times 10^{15}$ atoms $/ \mathrm{cm}^{3}$ was observed at $19 \mathrm{~K}, 18.5$ bar and with a beam diameter of approximately $10 \mathrm{~mm}$ in the scattering chamber.
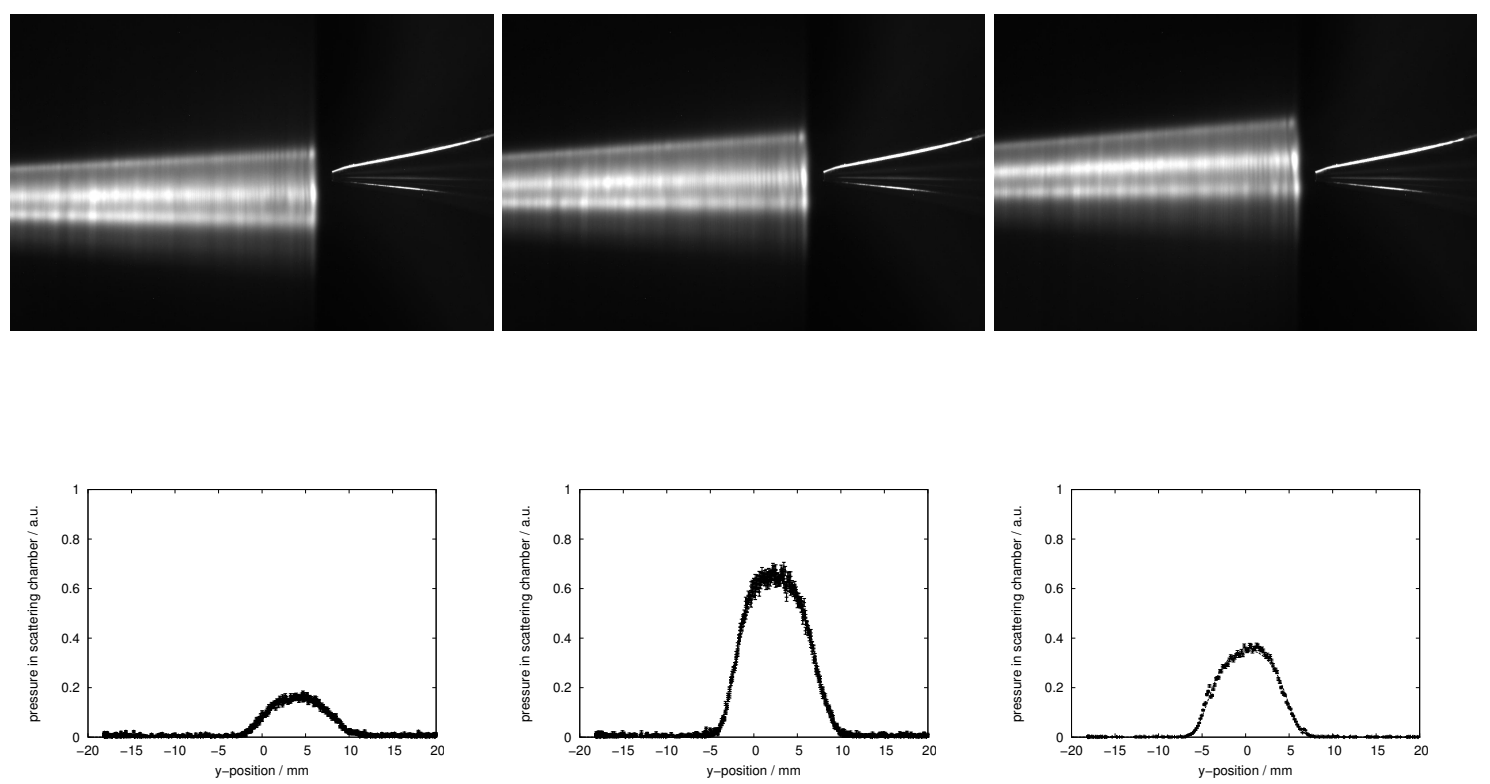

Figure 6: Photographs of the cluster beam in the skimmer chamber at constant hydrogen pressure and temperature settings of 18.5 bar and $19 \mathrm{~K}$. The use of the tilting system shows the change of the beam angle and the variation of the target density at the same pressure and temperature settings. The extraction of a bright part of the beam results in a higher density than a less bright part. 


\section{Improvement of vacuum conditions in the scattering chamber}

To reduce the influence of the cluster beam on the vacuum in the scattering chamber or rather in the HESR, first tests with special shaped collimators, were made. In Fig. 7 LM-micrographs of the used collimators one with, a round opening and one with a slit, are presented. The corresponding beam profiles at Fig. 8 show that the cluster beam is slightly shapeable with such an orifice. In this context systematic measurements to investigate the influence on the vacuum conditions and the target density are planned with the aim to determine the best collimator size.

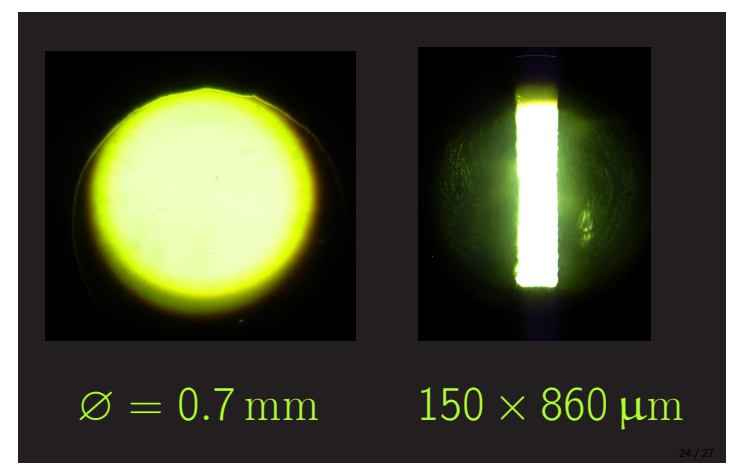

Figure 7: LM-micrographs of a collimator with a round opening and a slit. The collimator with a round opening has a diameter of $0.7 \mathrm{~mm}$. The slit collimator was produced with a special laser cut method and is in a dimension of $150 \times 860 \mu \mathrm{m}$.
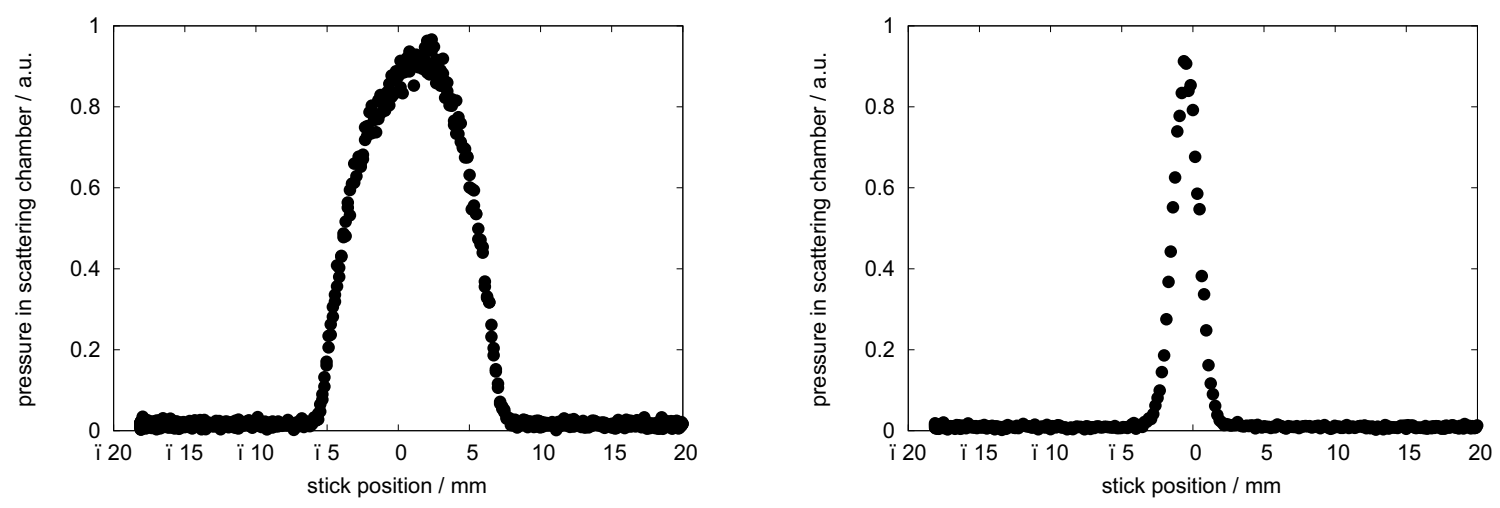

Figure 8: Cluster beam profiles with the use of a collimator of with a round opening (left) and a slit (right). The profiles show that the cluster beam is slightly shapeable. 


\section{Summary and Outlook}

A cluster-jet target prototype was designed and built up in complete PANDA-geometry. The observation of an inhomogeneous cluster-jet directly after the nozzle and the installation of a tilting system resulted in highest densities of $1.9 \times 10^{15}$ atoms $/ \mathrm{cm}^{3}$ and further improvements are expected. The prototype fulfils all mentioned requirements for $\overline{\mathrm{P}} \mathrm{ANDA}$. In next future systematic studies on the temperature and pressure settings with the highest density in combination with the tilting system are planned. Furthermore stability and reproducibility measurements will be performed. In addition feasibility studies on density adjustments in real time, e.g. with the variation of the pressure settings, the modification of the vacuum conditions at the skimmer chamber or the inception of wires/plates directly into the cluster beam are scheduled. Research on cluster mass and size are also intended as well as further tests with collimators with a slit. The construction of the final target for $\overline{\mathrm{P}} \mathrm{ANDA}$ is in progress.

\section{References}

[1] A. Khoukaz, Internal targets for the PANDA Experiment, in proceedings of STORI'11 conference, PoS (STORI11)036.

[2] A. Täschner, Velocity determination of hydrogen clusters at a cluster jet target, in proceedings of STORI'11 conference, PoS (STORI11)065.

[3] A. Täschner, Doctoral Thesis, Westfälische Wilhelms-Universität Münster, Germany (in preparation)

[4] A. Täschner et al., Nucl. Instr. and Meth. A 660 (2011) 22-30, doi:10.1016/j.nima.2011.09.024

[5] E. Köhler, Diploma Thesis, Westfälische Wilhelms-Universität Münster, Germany (2010) 